\title{
De la planification stratégique régionale à la prospective en Outaouais : la difficile construction d'un nouveau rapport au temps
}

\section{From strategic planning to regional foresight in the Outaouais:} The difficult construction of a new sense of time De la planificación estratégica regional, a la prospectiva en Outaouais: La difícil construcción de una nueva relación al tiempo

\author{
Martin ROBITAILLE, Guy CHIASSON et Mario GAUTHIER
}

Volume 60, numéro 170, septembre 2016

Prospective territoriale participative

Version originale soumise en mars 2016. Version révisée reçue en février 2017.

URI : https://id.erudit.org/iderudit/1040538ar

DOI : https://doi.org/10.7202/1040538ar

Aller au sommaire du numéro

Éditeur(s)

Département de géographie de l’Université Laval

ISSN

0007-9766 (imprimé)

1708-8968 (numérique)

Découvrir la revue

Citer cet article

ROBITAILLE, M., CHIASSON, G. \& GAUTHIER, M. (2016). De la planification stratégique régionale à la prospective en Outaouais : la difficile construction d'un nouveau rapport au temps. Cahiers de géographie du Québec, 60(170), 325-342. https://doi.org/10.7202/1040538ar
Résumé de l'article

À partir d'une analyse du cas Prospective Outaouais 2030, nous nous interrogeons sur les possibilités et les défis de la prospective dans le contexte d'une région dominée par une culture de planification stratégique régionale. Nous montrons tout d'abord que la planification stratégique et la prospective se démarquent sur le plan du rapport au temps : si la première privilégie des pratiques proches de l'action et inscrites dans le court terme, la seconde invite les acteurs du développement à se projeter à plus long terme. Ensuite, nous présentons les divers moments de la démarche suivie pour développer un exercice de prospective et une capacité de prise en compte du temps long dans la région de l'Outaouais. L'analyse de ces divers moments montre que la greffe d'une méthode prospective dans un terreau de planification stratégique régionale, même si elle est confrontée à des résistances, permet aussi d'ouvrir un dialogue et un espace de négociation en vue de construire, avec les acteurs, des scénarios inscrits dans le long terme sur la base d’une image partagée du territoire. 


\title{
De la planification stratégique régionale à la prospective en Outaouais: la difficile construction d'un nouveau rapport au temps
}

\author{
From strategic planning to regional foresight \\ in the Outaouais: The difficult construction of \\ a new sense of time
}

De la planificación estratégica regional, a la prospectiva en Outaouais: La difícil construcción de una nueva relación al tiempo

\author{
Martin ROBITAILLE, GuY CHIASSON et \\ Mario GAUTHIER \\ Université du Québec en Outaouais \\ Martin.Robitaille@uqo.ca \\ Guy.Chiasson@uqo.ca \\ Mario.Gauthier@uqo.ca
}

\section{Résumé}

À partir d'une analyse du cas Prospective Outaouais 2030, nous nous interrogeons sur les possibilités et les défis de la prospective dans le contexte d'une région dominée par une culture de planification stratégique régionale. Nous montrons tout d'abord que la planification stratégique et la prospective se démarquent sur le plan du rapport au temps : si la première privilégie des pratiques proches de l'action et inscrites dans le court terme, la seconde invite les acteurs du développement à se projeter à plus long terme. Ensuite, nous présentons les divers moments de la démarche suivie pour développer un exercice de prospective et une capacité de prise en compte du temps long dans la région de l'Outaouais. L'analyse de ces divers moments montre que la greffe d'une méthode prospective dans un terreau de planification stratégique régionale, même si elle est confrontée à des résistances, permet aussi d'ouvrir un dialogue et un espace de négociation en vue de construire, avec les acteurs, des scénarios inscrits dans le long terme sur la base d'une image partagée du territoire.

\section{Mots-clés}

Prospective régionale, planification stratégique, Outaouais, méthodologie prospective, région.

\begin{abstract}
Through a case study of Prospective Outaouais 2030, we reflect on the prospects and limits of a territorial prospective approach in a regional context dominated by a culture of strategic planning. First, we show how strategic planning and prospective methodologies differ in terms of their sense of time. Strategic planning tends to be close to action and typically refers to the short term while prospective methodologies usually prefer the long term. Secondly, we present the different steps that were taken during a prospective process in order to integrate the long term in the development of the Outaouais region. Our analysis shows how transplanting a prospective methodology in a context dominated by short-term planning, even while confronted by some resistance, allows for regional long-term scenarios to emerge.
\end{abstract}

\section{Keywords}

Regional prospective, strategic planning, Outaouais, prospective methodology, region. 


\section{Resumen}

A partir de un estudio de caso La prospectiva Outaouais 2030, investigamos posibilidades y desafíos de la prospectiva, en una región donde domina una cultura de planificación estratégica regional. Mostramos que la planificación estratégica y la prospectiva difieren en la relación al tiempo: mientras que la primera privilegia prácticas próximas a la acción e inscritas a corto plazo, la segunda, invita a los protagonistas a proyectarse a largo plazo. Luego, presentamos diversos momentos del proceso abordado para realizar un ejercicio de prospectiva y desarrollar la capacidad de considerar el largo tiempo, en la región del Outaouais. El análisis de las diversas etapas muestra que injertar un método prospectivo en un campo de planificación estratégica regional - aunque confronte oposiciones - permite entablar un dialogo y abrir un espacio de negociación para construir, con los protagonistas, escenarios inscritos a largo plazo, sobre una imagen compartida del territorio como base.

\section{Palabras claves}

Prospectiva regional, planificación estratégica, Outaouais, metodología prospectiva, región.

\section{Introduction}

Depuis les années 1960, la planification stratégique est devenue l'outil privilégié du développement régional, au Québec. Utilisée tant par les régions administratives que par les villes et les municipalités régionales de comté (MRC), cette méthode vise à faire le lien entre connaissance et action en combinant à la fois une visée stratégique fondée sur la formulation d'une vision commune et un objectif plus opérationnel de mise en œuvre de cette vision par des stratégies et des actions. Au fil du temps, la planification stratégique est devenue un espace d'efforts collectifs d'appropriation des enjeux territoriaux tant économiques que sociaux et culturels (Proulx, 2008) et a été appliquée aussi bien à des questions d'aménagement du territoire qu'à la gestion des politiques publiques ou des biens et des services, ou qu'au développement durable. En prenant appui sur un diagnostic territorial, elle a permis aux régions de développer des stratégies et de déterminer des actions à réaliser sur un temps court de quatre à cinq ans.

Alors que la planification stratégique était perçue au départ comme un exercice de planification autoritaire et technocratique (Proulx, 2014), les récentes évolutions du modèle en ont fait une approche plus collaborative, plus pragmatique et plus opérationnelle (Gauthier et al., 2008). Il n’en demeure pas moins que la planification stratégique régionale, au Québec, comporte certaines lacunes lorsque projetée sur le long terme. D’abord, les exercices de planification stratégique sont principalement sectoriels (santé et services sociaux, culture, enseignement, emploi, etc.) et ne contribuent que partiellement à une planification régionale d'ensemble. S’ajoutent à ces exercices des planifications économiques locales plus ciblées (agriculture, tourisme, foresterie, etc.) qui sont réalisées par les centres locaux de développement (CLD) et les sociétés d'aide au développement des collectivités (SADC) et qui morcellent encore plus les stratégies de développement. Par conséquent, la planification stratégique régionale a beaucoup de mal à s'imposer et à déboucher sur une vision d'ensemble du développement d'une région, d'autant plus qu'elle demeure indicative et sans pouvoirs contraignants et qu'elle se réalise avec peu de 
ressources, sans trop de participation ni de capacité d'agir sur le développement (Proulx, 2007). Ces exercices ont aussi tendance à cadrer fortement l'analyse dans un espace-temps de courte durée qui ne permet pas aux acteurs de penser leur développement autrement qu'en fonction des tendances lourdes, très ancrées dans l'histoire du territoire, et donc d'imaginer des futurs désirables et de faire émerger des scénarios différenciés de leur développement.

C'est à la suite d’un exercice de planification stratégique régionale (2012-2017) mené en Outaouais et ayant soulevé son lot d'insatisfactions que nous avons été amenés à nous intéresser à la prospective. Selon la définition proposée par Wachter dans le Dictionnaire de la géographie (Lévy et Lussault, 2003 : 749), la prospective territoriale ${ }^{1}$ est "une méthode d'exploration du futur" qui "cherche à sonder les devenirs possibles d'un territoire (région, ville, État, Europe...) tout en visant à éclairer des choix publics et à obtenir des résultats politiques ». Créée dans les années 1950 sous l'impulsion de la Délégation interministérielle à l'aménagement du territoire et à l'attractivité régionale (DATAR), la prospective s'est surtout développée en France à partir des années 1990, à la faveur de grands programmes nationaux qui ont encouragé la multiplication des expériences et aidé les territoires à mettre en place les outils et les dynamiques temporelles et collectives nécessaires (Decouflé, 2000a; Fourny et Denizot, 2008). Cette approche, qui part du principe que «l'avenir ne se prévoit pas, il se construit» (De Jouvenel, 1964), invite à considérer l'avenir non plus comme une chose décidée, mais comme une chose à faire.

Même si Godet (2007) voit des synergies potentielles entre les deux approches, peu de travaux ont permis jusqu'à maintenant d'éclairer les possibilités et les défis de l'implantation d'une méthodologie prospective dans un contexte territorial fortement imprégné par la planification stratégique régionale. Plusieurs questions nous interpellent dans cet article. Par exemple, comment sortir du cadre pragmatique, assez procédural et limité de la planification stratégique régionale? En quoi l'expérience française de prospective régionale peut-elle contribuer à enrichir la planification stratégique régionale au Québec? Enfin, comment permettre aux acteurs du développement d'une région de participer davantage à cette planification et d'imaginer des futurs souhaitables sur un temps plus long? Le meilleur des deux mondes résiderait-il dans une approche combinée de "prospective stratégique», comme l'affirment Godet et Durance (2011) ? Suivant l'intuition de ces auteurs, notre ambition dans ce texte est de voir dans quelle mesure la prospective régionale à la française (Wachter, 2003) peut enrichir la planification stratégique régionale telle qu'elle s'est institutionnalisée au Québec depuis plus de 30 ans. Plus précisément, nous voulons explorer deux hypothèses complémentaires. La première considère que la greffe d'une démarche prospective dans un contexte québécois dominé par la planification stratégique comporte des défis pratiques importants du fait d'un rapport au temps différent. La seconde hypothèse veut que, malgré les difficultés, le rapport au temps long permette de pallier certaines limites de la planification stratégique régionale à la québécoise.

1 La littérature parle de prospective territoriale ou de prospective régionale selon que le terme est employé dans son sens plus générique ou qu'il est appliqué à l'échelle d'une région. Dans ce texte, nous privilégions l'emploi du terme prospective régionale puisque notre propos porte principalement sur cette échelle territoriale. 
Dans la première partie du texte, nous amorçons cette réflexion en faisant ressortir l'historique et les particularités de la planification stratégique régionale au Québec, ainsi que les limites de cette approche. Nous explorons ensuite les spécificités de la prospective régionale en France, dans le but de voir comment cette approche pourrait élargir la portée de la planification stratégique au Québec en comblant certaines de ses lacunes. Plus particulièrement, nous cherchons à mettre en relief les distinctions entre les deux approches en ce qui a trait au rapport au temps. Dans la seconde partie du texte, nous revenons sur l'expérience de prospective régionale tentée dans la région québécoise de l'Outaouais pour illustrer, à travers un cas concret, la tension entre les temps longs et les temps courts vécue par les acteurs du développement. Une analyse critique de cet exercice nous amène enfin à mieux cerner les contraintes liées au contexte québécois et à considérer certaines pistes susceptibles de favoriser l'implantation d'une approche prospective comme prémisse à la planification territoriale au Québec.

\section{La planification stratégique régionale (PSR) au Québec}

Historiquement, la planification stratégique est issue du management stratégique de gestion dans les entreprises privées, qui remonte aux années 1960 (Martinet, 2008; Mintzberg et al., 2009). S’appuyant sur des visées théoriques et pratiques essentiellement liées à l'efficience et à l'efficacité des organisations, la planification stratégique est une approche qui amène l'organisation à réfléchir aux objectifs qu'elle veut atteindre, puis à déterminer les moyens à mettre en œuvre pour y parvenir en tenant compte de ses valeurs et de sa mission. Cette organisation est donc appelée à remettre ses objectifs en question par rapport à son environnement interne et externe, sur la base de ses forces et de ses faiblesses, ainsi que ses opportunités et les menaces auxquelles elle est confrontée (Martinet, 1983).

Dans la sphère publique québécoise, divers outils de management stratégique ont fait leur apparition au début des années 2000, après l'adoption de la Loi sur l'administration publique, qui obligeait tous les organismes publics à se doter d'une planification stratégique se déroulant sur un horizon de quatre à cinq ans. Aux niveaux régional et local, les pratiques de planification stratégique se différencient de celles des organisations publiques, en ce sens qu'elles ont été amorcées dès le début des années 1990 afin de moduler l'allocation des ressources publiques sur la base de stratégies régionales et locales (Proulx, 2008). Peu encadrés par la loi, ces exercices ont mené à des procédures régionales variables et à une multitude de planifications sectorielles et organisationnelles qui, dans les faits, ont occasionné beaucoup de confusion et une multiplication des stratégies de développement sur les territoires.

Pour le ministère des Affaires municipales et de l'Occupation du territoire (MAMOT), la planification stratégique régionale (PSR) représente «un processus de gestion visant à assurer, d'une manière continue, une concordance entre une organisation et les circonstances internes et externes qui lui sont favorables. Elle s'accomplit en adaptant la mission, en définissant des objectifs, en développant des stratégies et en élaborant un plan global qui indique comment les ressources seront utilisées pour répondre aux besoins des intéressés et aux exigences de l'environnement» (MAMOT, 2015). 
Essentiellement, le MAMOT demande que ces exercices se déroulent avec la participation de tous les intervenants (publics et privés) et des citoyens, selon une démarche continue, dynamique et interactive qui intègre tous les secteurs d'activité (tableau 1). Ces planifications doivent tenter de répondre à des questions spécifiques: où est-on aujourd'hui (le diagnostic)? où allons-nous (les tendances et les enjeux) ? où voulons-nous aller (la vision et les objectifs) ? comment allonsnous y arriver (les stratégies et les actions) ? (MAMOT, 2015).

\section{Tableau 1 Étapes de la planification stratégique au Québec}

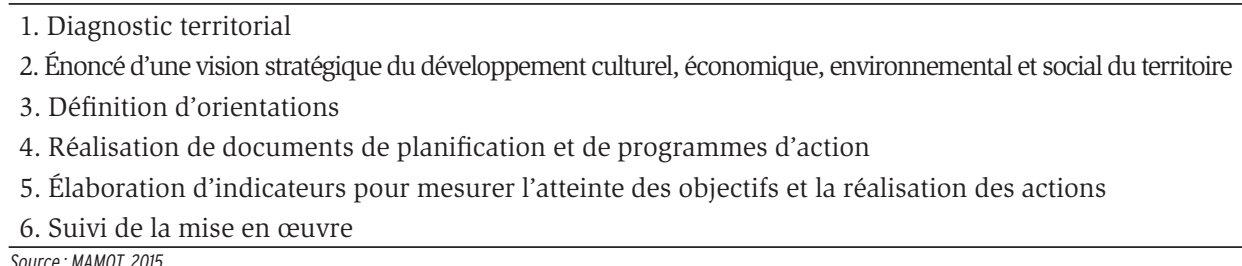

La définition et les étapes proposées de planification stratégique montrent bien l'importance accordée au caractère pragmatique et statique de la démarche, qui vise à gérer les besoins organisationnels et ceux des populations. La dimension prospective dans le processus en est absente, sinon par le biais d'un énoncé de vision stratégique qui peut certes reposer en partie sur le diagnostic territorial mais qui, dans les faits, se résume souvent à une vision souhaitée d'une région à long terme et ne repose pas sur un exercice de prospective à proprement parler.

L'absence de cadre législatif et normatif contraignant constitue par ailleurs une limite importante pour l'application de la PSR. Rappelons que, dans l'espace régional et local au Québec, il existe 17 régions administratives, $96 \mathrm{MRC}$ et de multiples organisations sectorielles (santé et services sociaux, emploi, tourisme, développement économique, culturel, etc.) qui ont à réaliser des planifications stratégiques d'une durée variable (quatre ou cinq ans) et s'échelonnant sur des périodes de temps tout aussi variables. Devant cette profusion de plans stratégiques, il devient impossible de dégager une vision commune à long terme reposant sur une étude sérieuse permettant d'anticiper le futur, de développer de véritables stratégies régionales qui permettent de contourner les intérêts sectoriels et corporatistes des organismes locaux et régionaux (Proulx, 2008) et d'évaluer les retombées réelles de ces planifications sur les territoires (VGQ, 2011). Selon Proulx (2007), la planification stratégique régionale demeure un exercice qui n'a pas les moyens de ses ambitions, car elle ne permet pas d'intégrer globalement les autres planifications existantes sur le territoire et d'en orienter les stratégies et les actions.

Cette forte tradition de planification stratégique régionale et locale, l'obligation imposée par le gouvernement du Québec de recourir à cet outil et la nature assez simple et peu contraignante du cadre d'application expliquent en partie le peu d'intérêt manifesté par les régions du Québec à l'égard de la prospective régionale. En fait, on recense seulement deux exercices québécois de prospective régionale, soit celui réalisé en 2004 au Saguenay-Lac-Saint-Jean à l'instigation de chercheurs de l'Université du Québec à Chicoutimi (Proulx, 2007) ainsi que celui réalisé en Outaouais, en 2014, avec le soutien des acteurs économiques de la région (Robitaille et al., 2015), qui sera décrit plus loin. 


\section{Les apports de la prospective régionale en France}

La réflexion menée en France sur les apports de la méthodologie prospective est riche d'enseignements sur les retombées potentielles de cette approche pour la planification stratégique régionale. En particulier, elle nous permet de mieux saisir comment la prospective contribue à inscrire le devenir des territoires dans une démarche permettant de concilier l'impératif stratégique du long terme à celui de la gestion du court terme, ce qui demeure un des défis à relever par la planification stratégique régionale au Québec.

La prospective, selon Loinger et Spohr (2005 : 34), «est une discipline, un ensemble de méthodes et plus largement une attitude qui vise à explorer les futurs d'un enjeu, d'un objet de la connaissance ou de l'action». Elle sert donc de support à l'activité de planification, qui demeure un cadre de référence pour l'action sur le territoire. Ainsi, la prospective territoriale ou régionale cherche des réponses à des questions centrales: quels futurs sont possibles? quelle société et quel territoire voulons-nous dans 20 ans? comment pouvons-nous les atteindre?

Qualifiée de «boule de cristal de l'aménagement du territoire» par Wachter (2000: 64), la prospective «vise à anticiper, à des horizons plus ou moins éloignés, les évolutions désirables ou non désirables d'un système» dans l'objectif de répondre à des problèmes de politique pratique. Elle constitue en ce sens «un outil de dialogue qui permet de confronter des choix et des stratégies» à partir de scénarios du futur élaborés dans le cadre de réflexions à long terme (Idem: 65), en vue de "produire un accord sur une image partagée d'un territoire à aménager» (Wachter, 2003: 750). La prospective consiste donc à penser sur le long terme, dans une démarche globale, et ainsi à agir avec plus d'efficacité sur les mécanismes de prise de décision à court et moyen termes. Elle favorise d'une part la créativité, mais aussi la déconstruction des représentations dominantes et enfin leur reconstruction, tout ceci dans un exercice collectif de «vision commune» (Berger, 1964).

Créée dans les années 1950 en France, la prospective a connu un premier âge d'or dans les années 1960 et 1970 sous l'impulsion de la DATAR (Decouflé, 2000a), avant d'être instaurée sur une plus grande échelle au début des années 1990, à la suite des premières lois de décentralisation. La prospective s'est alors répandue dans la majorité des régions françaises avec le soutien de programmes nationaux comme Territoires 2020, de 1990 à 1995, et Territoires 2030, à partir de 2004 (Fourny et Denizot, 2008). Les expériences nombreuses et diversifiées, soutenues par les conseils régionaux et/ou la préfecture des régions, avaient pour but de développer un schéma régional et de contribuer à l'élaboration du plan État-Région permettant l'accès à l'utilisation des fonds européens. Aux échelles infrarégionales (pays, agglomérations, etc.), la prospective s'est développée plus tardivement, avec l'objectif de déterminer les actions à entreprendre tout en contribuant à la construction de l'identité territoriale. Dans tous les cas de figure, la prospective demeure une méthode qui doit tenir compte du périmètre d'étude, des pouvoirs administratifs ou politiques et des compétences présentes sur les territoires, mais aussi de l'interdépendance des territoires, et cela, à toutes les échelles, de la commune à l'Europe (Delamarre, 2002).

En général, la prospective combine des travaux d'experts, des techniques de communication et des exercices de créativité. Elle est à l'articulation d'un univers qu'on peut situer dans le champ de la «gouvernance» du processus de projet territorial. Les méthodes développées par la prospective divergent de celles de la prévision et sont particulièrement adaptées au territoire: elles s'intéressent aux " temps longs » pour voir loin et large, et se veulent globales, brassant en même temps toutes les dimensions de la société: économiques, politiques, sociales, culturelles ou technologiques. En conséquence, ces méthodes sont à la fois qualitatives et quantitatives.

330 cahiers de géographie du Québec

Volume 60, numéro 170, septembre 2016 
La prospective ayant comme objectif de choisir une trajectoire parmi un ensemble de trajectoires possibles, elle repose sur l'élaboration de scénarios. On entend par scénario «la combinaison de séquences d’événements ou de phénomènes anticipés, ordinairement situées les unes par rapport aux autres dans un double système de relations diachroniques et causales, en vue de mettre en évidence les caractères probables de l'évolution d'une situation donnée, à partir d'un corps d'hypothèses formulées sur les "tendances lourdes" de cette évolution» (Decouflé, 2000b : 745-746). Ces scénarios peuvent être tendanciels ou contrastés. Les scénarios tendanciels sont le résultat d'une «simulation de processus d'évolutions possibles à partir d'une situation existante» alors que les scénarios contrastés ont pour objet d'élaborer "l'image future d'une situation donnée à partir d'hypothèses» (Idem: 746). Enfin, dans le champ de la prospective territoriale, on distingue généralement deux grands types : la prospective experte et la prospective participative. La première vise le développement d'une connaissance sur le processus de transformation du territoire et mobilise des spécialistes qui ont une expertise diversifiée dans différents domaines. La seconde produit une connaissance collective par des débats et des forums participatifs où se travaillent les représentations futures des territoires (Fourny et Denizot, 2008).

\section{Les temps de la prospective et de la planification stratégique régionale}

Comme nous l'avons vu précédemment, le temps de la planification stratégique régionale au Québec est relativement court (quatre à cinq ans), ce qui permet uniquement de fixer des objectifs à partir de la situation présente sur le territoire au lieu de susciter une mise à distance des habitudes et des héritages du passé (Bouzaiane et Mouelhi, 2008; Sgard, 2008). Ce faisant, la planification stratégique régionale écarte un ensemble de scénarios plausibles du futur pour se concentrer sur des stratégies et des actions répondant à des problématiques sectorielles ou de clientèles vécues par les entreprises et la population. Même si la planification stratégique régionale se réalise habituellement sur la base d'un diagnostic de la situation présente de la région, ce diagnostic repose surtout sur une analyse permettant de déterminer des éléments sur lesquels les acteurs du milieu doivent intervenir pour corriger des dysfonctionnements ponctuels.

La prospective régionale est donc une démarche permettant de produire une vision plus large que la planification stratégique régionale et qui permet de réfléchir sur le temps long (15 à 25 ans), soit l'horizon sur lequel portent les scénarios (figure 1). Selon Bouzaiane et Mouelhi (2008: 2), «[l]a prospective permet d'imaginer des situations nouvelles plus diversifiées et plus contrastées que ce que laissent dégager les techniques de prévision. Elle permet également d'avoir une vision des situations qui pourraient être plus intéressantes que des objectifs planifiés a priori et aussi de mettre en évidence des situations indésirables qui méritent d'être dévoilées suffisamment à l'avance».

Il n’en reste pas moins que les expériences françaises de prospective régionale ont démontré que l'engagement dans une réflexion, sur un temps long, demeure difficile tant pour les élus que pour les planificateurs, qui n'ont "pas le temps » et préfèrent souvent un moyen terme plus facile à imaginer et à planifier. Le tableau 2 ci-dessous montre comment les approches et les méthodes de la planification stratégique régionale et de la prospective régionale peuvent se différencier. Certes, le temps - ou l'horizon étudié - demeure un élément central de cette différence, mais les visées, la méthode et les finalités de ces démarches ne sont pas les mêmes. 


\section{Figure 1 Les temps de la prospective}

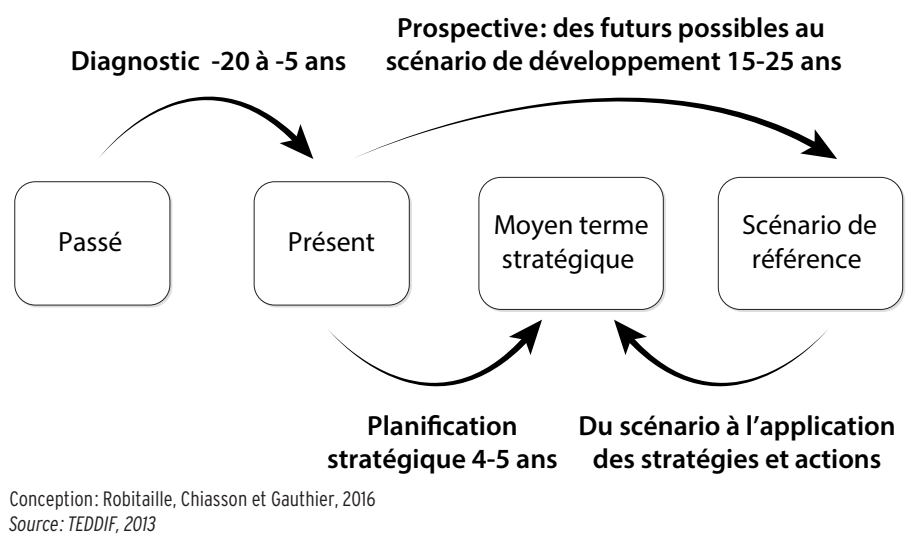

\section{Tableau 2 Comparaison de la planification stratégique régionale au Québec et de la prospective régionale en France}

\begin{tabular}{|c|c|c|}
\hline & $\begin{array}{c}\text { Planification stratégique } \\
\text { régionale au Québec }\end{array}$ & Prospective régionale en France \\
\hline $\begin{array}{l}\text { Visée de la } \\
\text { démarche }\end{array}$ & $\begin{array}{l}\text { - } \begin{array}{l}\text { Déductive et plus } \\
\text { pragmatique }\end{array} \\
\text { - } \text { Cherche à confirmer les } \\
\text { tendances lourdes } \\
\text { - Favorise une continuité des } \\
\text { politiques publiques }\end{array}$ & $\begin{array}{l}\text { - Inductive et plus intuitive } \\
\text { - Se penche sur les incertitudes } \\
\text { face à l'avenir } \\
\text { - Cherche à déceler les ruptures, } \\
\text { les signaux faibles } \\
\text { - Soulève des stratégies nouvelles }\end{array}$ \\
\hline Horizon & 4 à 5 ans & 15 à 25 ans \\
\hline Vision & $\begin{array}{l}\text { Implicite et basée sur un devenir } \\
\text { meilleur }\end{array}$ & $\begin{array}{l}\text { Plus explicite et construite } \\
\text { collectivement }\end{array}$ \\
\hline Diagnostic & $\begin{array}{l}\text { - Basé sur des études des } \\
\text { tendances du passé (5 à } 20 \text { ans) }\end{array}$ & $\begin{array}{l}\text { - Basé sur des études des tendances } \\
\text { du passé (5 à } 20 \text { ans) } \\
\text { - Nécessite un va-et-vient permanent } \\
\text { entre le diagnostic et la prospective }\end{array}$ \\
\hline Méthode & $\begin{array}{l}\text { - Basée sur des analyses } \\
\text { prévisionnelles et des besoins } \\
\text { présents sur le territoire }\end{array}$ & $\begin{array}{l}\text { - Basée sur l'exploration des } \\
\text { futurs possibles (construction de } \\
\text { scénarios) } \\
\text { - Nécessite des expertises } \\
\text { sectorielles et des itérations dans } \\
\text { les phases de la démarche }\end{array}$ \\
\hline Participation & $\begin{array}{l}\text { En aval de la démarche (débats } \\
\text { publics, forums, ateliers, etc.) }\end{array}$ & $\begin{array}{l}\text { En amont de la démarche (débats } \\
\text { publics, forums, ateliers, etc.) }\end{array}$ \\
\hline Stratégies & $\begin{array}{l}\text { Stratégies et actions à entreprendre } \\
\text { déterminées sur la base des } \\
\text { tendances lourdes et des besoins }\end{array}$ & $\begin{array}{l}\text { Choix stratégiques basés sur la vision } \\
\text { et les scénarios }\end{array}$ \\
\hline
\end{tabular}




\section{L'insertion d'une dimension prospective dans la planification stratégique régionale en Outaouais}

Le cas que nous analysons ici, Prospective Outaouais 2030, a été réalisé dans le cadre d'un partenariat entre des chercheurs de l'Université du Québec en Outaouais (UQO) et des acteurs de la région de l'Outaouais. Située au sud-ouest du Québec, cette région est l'une des 17 régions administratives du Québec. À l'instar des autres régions du Québec, son territoire est gouverné par trois paliers: le palier local des municipalités, le palier supralocal, avec cinq $\mathrm{MRC}^{2}$ et le palier régional, avec la Conférence régionale des élus de l'Outaouais (CREO). ${ }^{3}$ La particularité de la dynamique régionale de l'Outaouais vient du fait que la région est située à la frontière avec la province de l'Ontario, en particulier la Ville d'Ottawa, capitale nationale du Canada. Cette position frontalière influence à bien des égards la trajectoire de développement de la région et ne manque pas de façonner, au moins en partie, les efforts de prévision et de planification de l'avenir.

\section{L'Outaouais et son développement}

À compter du début du XIX ${ }^{\mathrm{e}}$ siècle, le développement de la région de l'Outaouais sera principalement axé sur sa vocation forestière (Gaffield, 1994). Ce n'est qu'à partir des années 1970 que l'économie des pâtes et papiers, qui a jusque-là dominé l'économie régionale, va faire place à une économie plus tertiaire prenant appui sur la fonction publique fédérale (Beaucage, 1994). Cette tertiarisation de l'économie urbaine va fortement contribuer à instaurer un développement régional à deux vitesses. D’une part, le pôle urbain et périurbain jouit d'une économie tertiaire dynamique, avec des salaires et un taux d'emploi plutôt élevés en raison d'une forte intégration avec la région métropolitaine d'Ottawa. D’autre part, dans les territoires plus éloignés du centre, la dépendance aux ressources naturelles reste bien présente, ce qui expose le développement des territoires aux soubresauts des marchés internationaux des matières premières (Chiasson, 2005).

\section{Le moment initial}

C’est dans ce contexte d'une région au développement fortement différencié entre le centre et la périphérie qu'a pris naissance l'initiative Prospective Outaouais 2030, dont la description ci-dessous est reprise en grande partie de Robitaille et al. (2015). De façon plus précise, cette initiative est également à situer en rapport

2 Au Québec, le palier MRC est gouverné par un conseil constitué d'élus locaux délégués par les municipalités locales. Lors de leur création, en 1979, les MRC avaient comme principale responsabilité l'aménagement du territoire. Depuis, plusieurs MRC ont étendu leur champ d’action à la suite d’un transfert volontaire de responsabilités des municipalités. L'Outaouais compte quatre territoires de MRC (Pontiac, Vallée de la Gatineau, Papineau et Collines de l'Outaouais) en plus de la Ville de Gatineau, qui a les responsabilités d'une MRC.

3 Les Conférences régionales des élus ont remplacé en 2003 les anciens Conseils régionaux de développement, à titre d'instance de concertation à l'échelle régionale. À l'automne 2014, alors que l'exercice de prospective en était à ses derniers moments, le gouvernement du Québec a annoncé l'abolition des Conférences régionales des élus (CRE) (Chiasson et Fortin, 2015). Comme nous le verrons plus loin, cette annonce a eu des effets sur notre démarche prospective. 
avec la planification stratégique régionale qui a été portée par la CREO. Cette planification, prévue pour l'horizon 2012-2017, faisait face à certaines critiques de la part d'acteurs de la région, particulièrement en ce qui a trait à l'incapacité de réfléchir à long terme sur un enjeu central, celui de la diversification économique dans un contexte de région frontalière avec l'Ontario fortement dépendante du secteur de l'administration publique fédérale. Ces insatisfactions vont donner lieu à la création, sous l'égide de la Chambre de commerce de Gatineau, d'un Conseil de développement économique régional de l'Outaouais (CDERO), qui sollicitera ensuite des chercheurs de l'UQO rattachés au Centre de recherche sur le développement territorial (CRDT) pour mettre à jour un portrait économique de la région réalisé quelques années plus tôt (Bensouda et Doucet, 2011). C'est sur la base de cette demande que nous avons proposé, à titre de chercheurs, d'élargir le mandat pour inclure un exercice de prospective devant aboutir à une vision de développement socioéconomique partagée par les acteurs-clés de la région. Cette vision était jugée nécessaire de part et d'autre pour répondre à certaines lacunes, tout en étant complémentaire à la planification stratégique régionale 2012-2017.

Les chercheurs et les partenaires du milieu se sont entendus au départ sur un exercice de prospective qui s'étalerait sur une période de six mois, c'est-à-dire de janvier à juin 2014, ce qui représente un défi en soi puisqu'un exercice prospectif prend généralement de 24 à 48 mois (Godet et Durance, 2011). Afin de rendre opérationnelle la démarche de prospective, trois instances de travail ont été mises sur pied : un comité de pilotage formé des membres de l'équipe de recherche de l'UQO et chargé d'assurer l'encadrement scientifique et méthodologique de la démarche, un comité de coordination regroupant l'équipe de recherche et les partenaires financiers du projet et chargé d'assurer le bon déroulement du projet et, finalement, un comité de réflexion stratégique, mis sur pied par le comité de coordination afin d'agir comme force motrice du travail de réflexion et de prospective devant aboutir à une vision commune du développement de la région de l'Outaouais sur l'horizon 2030. Le choix des membres de ce comité de réflexion était basé sur l'expertise que les individus pouvaient apporter à la démarche plutôt que sur leur appartenance à une institution ou à tel ou tel groupe incontournable, pour éviter que la réflexion soit trop rapidement balisée par des impératifs institutionnels et organisationnels. Sur ces bases, une trentaine d'intervenants ont été invités à participer et 15 experts ont accepté de se joindre au comité de réflexion. Comme en témoigne le tableau 3, le comité de réflexion stratégique initial était passablement diversifié, tant du point de vue du secteur d'activité que de la provenance territoriale. 
Tableau 3 Représentation des experts par secteur d'activité et par territoire dans le comité de réflexion stratégique

\begin{tabular}{l|c}
\hline Expertise et secteur d'activité & Territoire \\
\hline Élu (MRC) & Rural \\
Développement économique et entrepreneuriat & Rural \\
Développement économique des communautés & Rural \\
Municipalité & Rural \\
Emploi & Régional \\
Économie sociale & Régional \\
Développement social & Régional \\
Éducation postsecondaire & Régional \\
Développement économique & Régional \\
Santé et services sociaux & Urbain \\
Aménagement urbain & Urbain \\
Emploi & Urbain \\
Exportation & Urbain \\
Développement économique & Urbain \\
\hline Conception: Robitaille, chiasson et Gauthier, 2016 &
\end{tabular}

\section{Éléments de méthodologie pour construire le temps long}

Le comité de réflexion stratégique a tenu cinq réunions sur une période de huit mois, soit deux mois de plus que le calendrier prévu, pour compléter le travail d'élaboration du scénario qui a finalement été retenu: L'Outaouais 2030: une région unifiée et émancipée. Pour chacune de ces réunions, le travail de préparation fait par le comité de pilotage a été sensiblement le même: un cahier du participant préparé par l'équipe de recherche était acheminé aux participants une semaine à l'avance. Il contenait les principaux constats et résultats se dégageant de la réunion précédente, en plus des éléments d'information nécessaires pour le prochain atelier, incluant des rappels sur ce qu'est la méthode prospective. Bien que ne faisant pas nécessairement partie des outils de réalisation d'une prospective, les cahiers du participant se sont avérés un des points d'ancrage importants pour la bonne marche de l'exercice de prospective. La préparation de ces cahiers a exigé du comité de pilotage une somme de travail considérable, mais fructueuse, puisque les cahiers ont largement facilité le travail d'animation du comité de réflexion, en plus de laisser d'importants jalons de la démarche pour de futurs exercices de prospective.

Si les cahiers du participant ont permis d'assurer une certaine continuité entre les diverses réunions du comité de réflexion, les méthodes employées pour animer la réflexion et la discussion sur les objectifs visés ont varié de façon importante d'un atelier à l'autre. Le tableau 4 synthétise la trame méthodologique qui a guidé la mise en place des cinq ateliers. Les paragraphes qui suivent résument la contribution de chacun de ces ateliers à notre démarche. Une description plus étoffée de notre parcours méthodologique se trouve dans Robitaille et al. (2015). 


\section{Tableau 4 Trame méthodologique du travail de prospective en Outaouais}

\begin{tabular}{|c|c|c|}
\hline Cahiers du participant & Ateliers de prospective & Objectifs de l'atelier \\
\hline $\begin{array}{l}\text { Méthodologie } \\
\text { prospective } \\
\text { Diagnostic économique } \\
\text { et social } \\
\text { Synthèse de } \\
\text { planifications régionales } \\
\text { et locales actuelles }\end{array}$ & $\begin{array}{l}\text { ATELIER 1: Travail } \\
\text { d'imagination sur le futur } \\
\text { Comment imaginez-vous la } \\
\text { société de } 2030 \text { ? } \\
\text { Comment voyez-vous la région } \\
\text { de l'Outaouais en } 2030 \text { ? }\end{array}$ & $\begin{array}{l}\text { Synthèse des différents éléments de la } \\
\text { vision commune pour } 2030 \text { organisée } \\
\text { en } 5 \text { thématiques: capital économique, } \\
\text { capital politique, capital social et } \\
\text { identité, environnement et création de } \\
\text { richesse }\end{array}$ \\
\hline $\begin{array}{l}\text { Tendances mondiales, } \\
\text { nationales et } \\
\text { régionales: tendances } \\
\text { lourdes/tendances } \\
\text { légères }\end{array}$ & $\begin{array}{l}\text { ATELIER 2: Appréhension des } \\
\text { ruptures possibles } \\
\text { Que peut-il advenir? } \\
\text { Détermination des opportunités } \\
\text { et des menaces } \\
\text { Quelles sont les opportunités et } \\
\text { menaces pour la région? }\end{array}$ & $\begin{array}{l}\text { Tableau synthèse autour de quatre } \\
\text { thématiques: population et mode } \\
\text { de vie, développement économique, } \\
\text { environnement et gestion de l'espace, rôle } \\
\text { politique en société } \\
\text { Découpage des thématiques retenues } \\
\text { en } 20 \text { variables-clés }\end{array}$ \\
\hline $\begin{array}{l}\text { Tableau de méthode } \\
\text { d'analyse structurelle } \\
\text { MicMac }\end{array}$ & $\begin{array}{l}\text { ATELIER 3: Travail d'interrelation } \\
\text { des } 20 \text { variables } \\
\text { Quelle est l'influence de la } \\
\text { variable X sur la variable Y? } \\
\text { Attribution de poids pour } \\
\text { chaque variable } \\
\text { Quelle est l'importance de la } \\
\text { variable X pour le développement } \\
\text { régional? }\end{array}$ & $\begin{array}{l}\text { Détermination des variables pour } \\
\text { l'Outaouais: } \\
\text { (1) internes/externes } \\
\text { (2) motrices/dépendantes/relais et } \\
\text { autonomes }\end{array}$ \\
\hline $\begin{array}{l}\text { Deux scénarios aux } \\
\text { dynamiques distinctes }\end{array}$ & $\begin{array}{l}\text { ATELIER 4: Débat autour du } \\
\text { «futur possible» et du «futur } \\
\text { probable» } \\
\text { Parmi les deux scénarios, quel } \\
\text { est le plus probable? Le plus } \\
\text { souhaitable? } \\
\text { Pour chaque scénario, quels sont } \\
\text { les éléments les plus probables? Les } \\
\text { plus souhaitables? }\end{array}$ & $\begin{array}{l}\text { Détermination des éléments les plus } \\
\text { probables et les plus souhaitables des } \\
\text { scénarios }\end{array}$ \\
\hline $\begin{array}{l}\text { Scénario Outaouais } \\
2030 \\
\text { Documents diagnostics } \\
2014\end{array}$ & $\begin{array}{l}\text { ATELIER 5: Établissement d'un } \\
\text { consensus autour d'un troisième } \\
\text { scénario } \\
\text { Travail de projection } \\
\text { Quels seraient les éléments, } \\
\text { dans votre travail quotidien, qui } \\
\text { iraient dans le sens de la vision } \\
\text { Outaouais 2030? }\end{array}$ & $\begin{array}{l}\text { Détermination du scénario L'Outaouais } \\
\text { 2030: une région unifiée et émancipée } \\
\text { Prémisse de réflexions sur (1) les atouts } \\
\text { dont on s'est doté et (2) les freins actuels } \\
\text { pour parvenir au scénario } \\
\text { Rapport final sur l'exercice de } \\
\text { prospective }\end{array}$ \\
\hline
\end{tabular}

Le premier atelier visait à établir, auprès des acteurs, l'intérêt d'un exercice de prospective et d'une réflexion sur le temps long dans le contexte régional, ce qui a été fait par l'entremise d'une présentation sur ce qu'est la prospective et d'une mise en perspective des limites des diverses planifications stratégiques établies sur le territoire régional. 
Lors du second et du troisième atelier, les acteurs ont été invités à faire un travail de détermination et de priorisation des variables qui influencent le développement de la région. Dans un premier temps (atelier 2), les acteurs, à partir de 15 variables initiales, ont cerné les tendances lourdes régionales et les ruptures possibles, ainsi que les menaces et opportunités que représentent ces tendances. Les tendances déterminées par le comité de réflexion ont ensuite été synthétisées en quatre grandes tendances qui se sont retrouvées au cœur des discussions de l'atelier 3, où nous avons fait appel à la méthode MicMac (Matrice d'impacts croisés Multiplication appliquée à un classement) (Godet et Durance, 2011). Rappelons que cette méthode vise à analyser les variables-clés d'un système, particulièrement celles porteuses d'enjeux pour son évolution, de façon à mettre en évidence (hiérarchiser) les variables influentes (ou motrices) et dépendantes. Pour ce faire, on utilisera une matrice pour déterminer le degré de motricité (capacité à influencer le développement) et le degré de dépendance de chaque variable, les unes par rapport aux autres. Finalement, les ateliers 4 et 5 ont été consacrés à la définition de scénarios pour le développement de la région sur un horizon 2030. Lors de l'atelier 4, des groupes de discussion ont eu à se prononcer sur deux scénarios contrastés : ${ }^{4}$ le premier, dont l'essor reposait sur deux dynamiques distinctes entre la partie métropolitaine et les MRC rurales, et le second, dans lequel la région de l'Outaouais était unifiée dans et pour son développement. Les groupes de discussion avaient à se prononcer, dans un premier temps, sur la "probabilité» et, dans un second temps, sur la «souhaitabilité» que les deux scénarios se réalisent. Ces scénarios se voulaient l'aboutissement du travail de clarification et de priorisation des tendances réalisé dans les ateliers précédents, de façon à dégager une vision commune d'un futur à la fois possible et souhaitable pour la région de l'Outaouais en 2030. De l'avis des participants, il était clair que le second scénario, celui qui prévoit un développement cohérent entre le pôle urbain et les MRC rurales, était à la fois plus probable et plus souhaitable. Cependant, le groupe de discussion n'était pas prêt à rejeter entièrement le premier scénario, considérant que certains de ses éléments méritaient d'être intégrés au scénario final. Dans ce contexte, les acteurs ont demandé la tenue d'une cinquième rencontre afin de valider un troisième scénario intégrant ces éléments. Ce dernier scénario a fait assez rapidement consensus lors de cette dernière rencontre (tableau 5). À des fins d'appropriation de ce scénario, les participants ont été amenés à débattre, après réflexion, des «éléments actuels qui vont dans le sens de la vision Outaouais 2030» et des «freins qui pourraient retarder le développement de ce scénario».

\section{Tableau 5 Principaux éléments du scénario L'Outaouais 2030: une région unifiée et émancipée}

\begin{tabular}{l|l}
\hline \multicolumn{1}{c}{$\begin{array}{c}\text { Vision d'une région unifiée ouverte à des stratégies de complémentarité rurale-urbaine } \\
\text { permettant d'atténuer les conflits de l'espace urbain ou rural }\end{array}$} \\
\hline $\begin{array}{l}\text { La contribution de } \\
\text { la région urbaine de } \\
\text { l'Outaouais }\end{array}$ & $\begin{array}{l}\text { Une économie qui repose sur l'innovation, le savoir et les compétences } \\
\text { Une diversification économique basée sur les forces du milieu et des } \\
\text { alliances } \\
\text { Un développement des établissements d'enseignement supérieur } \\
\text { Un pôle urbain qui mise sur le développement durable }\end{array}$ \\
\hline $\begin{array}{l}\text { La contribution des } \\
\text { territoires ruraux de } \\
\text { l'Outaouais }\end{array}$ & $\begin{array}{l}\text { Un développement économique complémentaire entre les MRC rurales } \\
\text { Une attractivité accrue (compétitivité économique et infrastructures } \\
\text { numériques) } \\
\text { Une plus grande cohérence (collaboration/coopération) dans un objectif de } \\
\text { complémentarité des MRC }\end{array}$ \\
\hline
\end{tabular}

Conception: Robitaille, Chiasson et Gauthier, 2016

4 Des scénarios contrastés, tel qu'on les utilise en prospective, supposent des configurations morphologiquement différentes. Ce n'est pas un peu plus ou un peu moins la même chose, mais une autre histoire bâtie à partir de transformations structurelles du système. 


\section{Les difficultés: insérer le temps long dans une démarche courte}

Nous avons suivi, parfois avec des adaptations, la séquence habituelle pour un travail de prospective. Globalement, on peut considérer que cette démarche a porté des fruits dans le sens où la mise en pratique de la méthodologie prospective a effectivement permis de se doter d'une compréhension commune des avenirs possibles de la région, au-delà des horizons habituels de la planification stratégique. Par contre, la greffe d'une méthodologie prospective à une planification stratégique régionale ne se fait pas sans heurts, comme le démontre la section qui suit.

On remarquera d'abord que l'exercice de prospective a été mené dans un cadre plus ou moins propice, et cela, autant pour le calendrier que pour le financement. Malgré qu'une étude prospective régionale prenne souvent plus d'une année à réaliser, le comité de coordination a tout de même retenu un calendrier de travail très serré de huit mois. Ce calendrier était d'autant plus restrictif que les sommes consenties au projet sous-estimaient de façon assez importante la quantité de travail nécessaire au déploiement de cette méthodologie. Ainsi, plusieurs étapes du projet n'ont pu aboutir qu'avec l'apport de ressources supplémentaires assez importantes du CRDT et au prix d'un travail considérable, réalisé souvent dans l'urgence et sans nécessairement la capacité de recul nécessaire à une telle activité. Sans doute par manque d'expérience en prospective, les acteurs impliqués dans la direction du projet, incluant les chercheurs, ont nettement sous-estimé l'effort collectif à consentir pour se doter d'une réelle capacité d'intégrer une vision de référence partagée dans la planification stratégique régionale.

Le délai requis pour construire une capacité de prise en compte du temps long avec le comité de réflexion a parfois fait l'objet de discussions animées au comité de coordination. Les acteurs, partenaires du projet, ont parfois montré des signes d’impatience face à une démarche qui prenait du temps à aboutir. À plusieurs reprises, le comité de pilotage a dû rappeler l'importance, dans une démarche prospective, de laisser le temps à un parcours collectif d'appropriation du futur de suivre son cours. Ces discussions ont été particulièrement manifestes à partir du moment où le projet de forum socioéconomique s'est concrétisé, ce qui a augmenté la pression pour que la démarche «livre la marchandise» et donc qu'on réinscrive le processus dans l'action. Ces discussions, qui ont passablement compliqué le travail de coordination de la démarche, nous semblent assez révélatrices d'une certaine habitude de la planification stratégique régionale chez les acteurs de l'Outaouais et de la nécessité, dans ce contexte, de rappeler l'importance de se donner les moyens d'incorporer le temps long.

La différence de culture entre la planification stratégique régionale et la prospective régionale s'est également manifestée à l'intérieur du comité de réflexion stratégique lui-même. Rappelons que le comité de coordination avait sélectionné des gens sur la base de leur expertise particulière plutôt que de leur appartenance à telle ou telle organisation, et il a pris soin de rappeler aux participants qu'ils étaient interpelés à ce titre. Le but était de s'éloigner de la logique organisationnelle proche de l'action, qui est dominante dans la planification stratégique régionale, pour permettre aux participants d'imaginer ensemble les futurs de la région sans être trop contraints dans le présent. La logique organisationnelle a toutefois fait un certain retour dans le comité de réflexion, surtout lors des discussions sur les scénarios dans les derniers ateliers alors que des impératifs institutionnels ont refait surface dans le discours de certains acteurs tentés d'user de leur position d'autorité pour dicter un peu 
l'orientation des travaux du comité. Cette dynamique a sûrement été renforcée par le climat d'incertitude croissante qui régnait à l'automne 2014 au moment où les plans du gouvernement québécois de transformer le paysage institutionnel des régions québécoises commençaient à circuler. Les organisations représentées autour de la table étant toutes touchées d'une façon ou d'une autre par les changements à venir, le contexte politique a semblé favoriser une certaine crispation dans la défense des intérêts chez certains et entraîné une fluctuation des acteurs présents lors des derniers ateliers. Ces éléments ont contribué à rendre le climat moins propice à la prospective.

\section{L'aboutissement: le Forum socioéconomique de l'Outaouais}

À mi-parcours de la démarche de prospective, les partenaires ont proposé que les scénarios élaborés dans le cadre de cette démarche soient rendus publics dans le cadre d'un forum socioéconomique. Ce forum, finalement tenu le 29 novembre 2014, représentait en quelque sorte l'aboutissement de notre démarche et une fenêtre privilégiée pour examiner le rapprochement entre la prospective régionale et la planification stratégique régionale.

Organisé par les chercheurs de l'UQO à l'instigation de la Chambre de commerce de Gatineau, le Forum a réuni 150 personnes qui ont été conviées à déterminer les actions à prendre pour favoriser une trajectoire régionale alignée avec la vision inscrite dans le scénario retenu. En guise de préparation, le scénario Outaouais 2030 avait circulé largement auprès des acteurs du développement de la région pour leur donner l'occasion de réfléchir à des actions à prendre pour le mettre en œuvre. Plus de trente acteurs régionaux ont fait parvenir des réactions qui ont ensuite été compilées et présentées lors du Forum.

L'intérêt suscité par le Forum auprès des acteurs du développement de la région et dans les médias régionaux, tout comme la volonté du CDERO d'en faire un événement public à grand déploiement, nous semblent être des indicateurs d'un certain appétit régional pour le développement d'une vision et d'un scénario à long terme. Interpelés pour la première fois sur un futur possible, les acteurs ont manifestement apprécié cet exercice qui sortait du cadre traditionnel (et insatisfaisant) de la planification stratégique. À l’issue du Forum, le scénario développé à la suite du travail du comité de réflexion semblait d'ailleurs faire consensus auprès d'un assez large public d'acteurs engagés.

Cependant, un peu plus de deux ans après l'événement, force est de constater que l'exercice a eu peu de suites et que la prise de conscience collective qu'il a suscitée semble s'estomper. Or, cet effort inédit de mobilisation des acteurs autour d'un projet d'élaboration d'une vision à long terme pour la région de l'Outaouais aurait pu avoir un impact réel, n'eût été l'abolition des conférences régionales des élus dans la foulée des mesures d'austérité adoptées par l'État québécois. En effet, avec la disparition de la CREO, l'instance chargée d'animer et de mettre en place la PSR, il n'existe plus vraiment d'instance de concertation à l'échelle régionale pour porter la planification, pas plus qu'il n’y a de support institutionnel régional auquel raccrocher la vision du développement élaborée dans le cadre de l'exercice de prospective. Il devient donc difficile, dans ce contexte, d'amener les acteurs du développement socioéconomique à travailler de concert pour la mettre en œuvre, d'autant plus qu'en l'absence de conditions propices, les forces traditionnelles prennent le pas rapidement sur toute velléité de changement. À cet égard, et comme l'ont souligné les experts et les participants au Forum, on peut conclure que l'intervention de l'État ou, dans ce cas-ci, le désengagement de l'État, constitue la première variable motrice de développement de la région de l'Outaouais. 


\section{Conclusion}

Cet article a porté sur la complémentarité entre la prospective régionale et la planification stratégique régionale. Ces deux méthodes renvoient à des rapports singuliers au temps qui sont imprégnés dans les pratiques et les institutions des territoires. L'analyse de l'exercice Prospective Outaouais 2030 a permis de voir que devant cette "différenciation territoriale», liée à la mobilisation des acteurs et aux temps d'action des politiques publiques, la greffe d'une approche prospective dans un contexte territorial plus habitué à une temporalité de planification stratégique ne va pas toujours de soi. Cela est attribuable à une visée très pragmatique de ces exercices de planification stratégique régionale qui sont renouvelables tous les cinq ans et qui servent essentiellement de suivi d'allocation des ressources.

À l'instar de l'expérience tentée en Outaouais, la démarche prospective participative du Saguenay-Lac-Saint-Jean, Vision 2025, n’a pas non plus donné les retombées espérées. Perçue par la communauté comme un exercice issu du milieu universitaire, cette première expérience de prospective à la québécoise n’a pas débouché sur la mise en place d'un mécanisme (forum) capable de porter cette vision, et les connaissances acquises n’ont pas suffisamment percolé dans les planifications stratégiques des organismes du développement de la région (Proulx, 2007).

Dans le cas de Prospective Outaouais 2030, le dispositif méthodologique mis en place visait à accompagner les acteurs vers le développement d'une capacité de prise en compte du temps long dans la réflexion sur l'avenir de la région. Or, la démarche s'est heurtée à de nombreux freins (ressources limitées, un certain désengagement des participants, contraintes de temps pour la réflexion et pour l'action, etc.) et à un contexte de mise en œuvre peu propice, qui témoignent d'une certaine résistance des acteurs et des institutions du milieu. Cela suggère que l'intégration du temps long dans des territoires de planification stratégique nécessite un changement de culture organisationnelle, plus de ressources et, surtout, un patient travail de construction.

L'analyse des divers moments de la démarche illustre par ailleurs que la greffe d'une méthode prospective dans un terreau de planification stratégique régionale, bien que confrontée à des résistances, a tout de même porté des fruits intéressants - comme le développement d'une vision s'appuyant sur l'intégration du temps long - qui démontrent le potentiel de la prospective dans le contexte des territoires québécois. Le Forum socioéconomique, en tant qu'exercice de prospective participative, a en ce sens permis d'ouvrir un dialogue et un espace de négociation en vue d'aider à déconstruire les représentations dominantes pour construire, avec les acteurs, de nouveaux scénarios inscrits dans le long terme sur la base d'une image partagée du territoire. Les changements apportés par le gouvernement québécois aux institutions régionales et la remise en question plus globale de la planification stratégique régionale comme mécanisme de développement régional rendent cependant beaucoup plus incertaine la mise en œuvre de ces visions. 


\section{Bibliographie}

BEAUCAGE, André (1994) De la manufacture aux services. Dans Chad Gaffield (dir.) Histoire de l'Outaouais. Québec, Institut québécois de recherche sur la culture, p. 493-540.

BENSOUDA, Reda et DOUCET, Chantale (2011) Portrait économique de l'Outaouais. Gatineau, Cahier de l'Alliance de recherche université-communauté en Innovation sociale et développement des communautés et Université du Québec en Outaouais.

BERGER, Gaston (1964) L'attitude prospective. Phénoménologie du temps et prospective. Paris, Presses Universitaires de France.

BOUZAIANE, Lotfi et MOUELHI, Rim (2008) Introduction au concept de base de la prospective: projet de M2PA. Tunis, Université virtuelle de Tunis.

CHIASSON, Guy (2005) L'attractivité des territoires dans un contexte de mondialisation: quel espoir pour les milieux ruraux de l'Outaouais? Dans Jean-Charles Lollier, Lionel Prigent et Hervé Thouement (dir.) Les nouveaux facteurs d'attractivité dans le jeu de la mondialisation. Rennes, Presses Universitaires de Rennes, p. 131-142.

CHIASSON, Guy et FORTIN, Marie-Josée (2015) L'austérité comme laboratoire de restructuration des rapports entre l'État et les territoires. Organisations et territoires, vol. 24, no3, p. 3-6.

DE JOUVENEL, Bertrand (1964) L'art de la conjecture. Paris, Éditions du Rocher.

DECOUfLÉ, André-Clément (2000a) Prospective. Dans Pierre Merlin et Françoise Choay (dir.) Dictionnaire de l'urbanisme et de l'aménagement. Paris, Presses Universitaires de France, p. 686.

DECOUflé, André-Clément (2000b) Scénario. Dans Pierre Merlin et Françoise Choay (dir.) Dictionnaire de l'urbanisme et de l'aménagement. Paris, Presses Universitaires de France, p. 745-747.
DELAMARRE, Aliette (2002) La prospective territoriale. Paris, La Documentation française et la Délégation interministérielle à l'aménagement du territoire et à l'attractivité régionale.

FOURNY, Marie-Christine et DENIZOT, Damien (2008) La prospective territoriale, révélateur et outil d'une action publique territorialisée. Dans Rodolphe Dodier, Alice Rouyer et Raymonde Séchet (dir.) Territoires en action et dans l'action. Rennes, Presses Universitaires de Rennes, p. 29-43.

GAFFIELD, Chad (dir.) (1994) Histoire de l'Outaouais. Québec, Institut québécois de recherche sur la culture.

GAUTHIER, Mario, GARIÉPY, Michel et TRÉPANIER, Marie-Odile (dir.) (2008) Renouveler l'aménagement et l'urbanisme: planification territoriale, débat public et développement durable. Montréal, Les Presses de l'Université de Montréal.

GODET, Michel (2007) Manuel de prospective stratégique. Paris, Éditions Dunod.

GODET, Michel et DURANCE, Philippe (2011) La prospective stratégique pour les entreprises et les territoires. Paris, Éditions Dunod, l'Organisation des Nations unies pour l'éducation, la science et la culture et la Fondation Prospective et Innovation.

LÉVY, Jacques et LUSSAULT, Michel (dir.) (2003) Dictionnaire de la géographie et de l'espace des sociétés. Paris, Belin.

LOINGER, Guy et SPOHR, Claude (2005) Prospectives et planification territoriales: état des lieux et propositions. Travaux de recherche de prospective, no24, p. 1-198.

MAMOT (MINISTÈRE DES AFFAIRES MUNICIPALES ET DE L'OCCUPATION DU TERRITOIRE) (2015) Guide La prise de décision en urbanisme. Outils de planification [En ligne]. http://www. mamrot.gouv.qc.ca/amenagementdu-territoire/guide-la-prise-dedecision-en-urbanisme/planification/ planification-strategique/ 
MARTINET, Alain-Charles (1983) Les procédures de la planification. Stratégie. Paris, Éditions Vuibert.

MARTINET, Alain-Charles (2008). 50 ans de recherche en stratégie: normalisation ou pluralisme épistémologique? Finance Contrôle Stratégie. vol.11, p. 39-66.

MINTZBERG, Henry, AHLSTRAND, Bruce et LAMPEL, Joseph (2009) Safari en pays stratégie: l'exploration des grands courants de la pensée stratégique. Paris, Pearson Education France.

PROULX, Marc-Urbain (2007) Vision 2025: le Saguenay-Lac-Saint-Jean face à son avenir. Québec, Presses de l'Université du Québec.

PROULX, Marc-Urbain (2008) Quatre décennies de planification territoriale au Québec. Dans Mario Gauthier, Michel Gariépy et Marie-Odile Trépanier (dir.) Renouveler l'aménagement et l'urbanisme. Montréal, Presses de l'Université de Montréal, p. 23-54.

PROULX, Marc-Urbain (2014) Saisir la pratique québécoise de planification territoriale. Dans Martin Robitaille et Marc-Urbain Proulx (dir.) Sciences du territoire: défis méthodologiques. Québec, Presses de l'Université du Québec, p. 365-386.

ROBITAILLE, Martin, PLASSIN, Élodie et COCHARD, Amandine (2015) L'Outaouais en prospective territoriale. Organisation et territoires, vol.24, n² 2, p. 13-18.

SGARD, Anne (2008) Entre rétrospective et prospective. Revue disciplinaire des sciences sociales - Espacestemps.net [En ligne]. http://www.espacestemps.net/ articles/entre-retrospective-et-prospective/

TEDDIF (TERRITOIRES, ENVIRONNEMENT ET DÉVELOPPEMENT DURABLE EN ÎLE-DE-FRANCE) (2013) La prospective appliquée aux projets territoriaux de développement durable. Les cahiers $d u$ développement durable en Île-de-France, $\mathrm{n}^{\mathrm{0}} 10$, p. 1-40.

VGQ (VÉRIFICATEUR GÉNÉRAL DU QUÉBEC) (2011) Rapport du commissaire au développement durable - Rapport du Vérificateur général du Québec à l'Assemblée nationale pour l'année 20112012. Québec, Gouvernement du Québec.
WACHTER, Serge (2000) Prospective. Dans Serge Wachter, Alain Bourdin, Jacques Lévy, Jean-Marc Offner, Jean-Gustave Padioleau, Franck Scherrer et Jacques Theys (dir.) Repenser le territoire. Un dictionnaire critique. La Tour d'Aigues, Éditions de l'Aube et Délégation interministérielle à l'aménagement du territoire et à l'attractivité régionale.

WACHTER, Serge (2003) Prospective territoriale. Dans Jacques Lévy et Michel Lussault (dir.) Dictionnaire de la géographie et de l'espace des sociétés. Paris, Belin, p. 749-750. 